\title{
Rhabdoid tumors in children: Prognostic factors in 70 patients diagnosed in Germany
}

\author{
HARALD REINHARD $^{1}$, JENNIFER REINERT ${ }^{1}$, RITA BEIER ${ }^{1}$, RHOIKOS FURTW ÄNGLER ${ }^{1}$, \\ MUHANAT ALKASSER ${ }^{1}$, STEFAN RUTKOWSKI ${ }^{2}$, MICHAEL FRÜHWALD ${ }^{3}$, \\ EWA KOSCIELNIAK ${ }^{4}$, IVO LEUSCHNER ${ }^{5}$, PETER KAATSCH ${ }^{6}$ and NORBERT GRAF ${ }^{1}$
}

\begin{abstract}
${ }^{1}$ Department of Pediatric Hematology and Oncology, University Hospital Homburg/Saar; ${ }^{2}$ Department of Pediatric Hematology and Oncology, University Hospital Würzburg; ${ }^{3}$ Department of Pediatric Hematology and Oncology, University Hospital Münster; ${ }^{4}$ Department of Pediatric Hematology and Oncology, Olgahospital, Stuttgart; ${ }^{5}$ Department of Paidopathology, University Hospital Kiel; ${ }^{6}$ German Childhood Cancer Registry (GCCR), Institute of Medical Biostatistics, Epidemiology and Informatics (IMBEI), University of Mainz, Germany
\end{abstract}

Received August 30, 2007; Accepted November 9, 2007

\begin{abstract}
We retrospectively analysed the data of 70 patients (41 boys and 29 girls) with Rhabdoid tumors (RT) regardless of localisation, recorded in the German Childhood Cancer Registry (GCCR) from 1984 to 1999 . The primary tumor was located in the kidney in 32 cases, in the central nervous system (CNS) in 13 cases and in the soft tissue in 25 cases. Variables examined were tumor stage, sex, age at diagnosis, surgical radicality, radiotherapy and chemotherapeutic regimens. Metastatic disease at diagnosis was observed in 18 of the 70 individuals. Outcome of this group was very poor with a 5-year overall survival of $11 \%$. There were no differences in survival between males and females, or younger and older children. Chemotherapeutic regimens were mainly given according to the primary site of the tumor. Radiotherapy was given in 28 of the 70 patients with a mean dose of 35 Gray, though this did not improve the outcome. Overall survival of the whole cohort was $27 \%$ at 5 years and there was no significant difference in prognosis regarding the different locations of the tumor (kidney 24\%, soft tissue 30\%, CNS 29\%). In conclusion, $\mathrm{RT}$ in infants and children has a dismal prognosis, independent from localisation. The presence of metastasis at diagnosis seems to be the only prognostic factor of outcome.
\end{abstract}

\section{Introduction}

Rhabdoid tumors (RT) in children and infants is a rare and highly aggressive malignancy. The tumor was classified as a

Correspondence to: Dr Harald Reinhard, Pediatric Hematology and Oncology, University Hospital, Building No 9, D-66421 Homburg/ Saar, Germany

E-mail: harald.reinhard@uniklinikum-saarland.de

Key words: Rhabdoid tumor, children, incidence, registry, prognostic factors distinct tumor entity in 1978 (1) and the tissue origin of RT is not yet clear. Cytogenetic analysis demonstrated monosomy 22 or loss of a band in 22q11 (2). Molecular genetics revealed a mutation of the SMARCB1/hSNF5/INI1 gene, which can be helpful for confirmation of diagnosis (3). The primary tumor can occur in different localisations and the main manifestations are in the kidney, soft tissue and central nervous system (CNS). In the CNS, it is commonly called Atypical Teratoid/ Rhabdoid tumors (AT/RT). The malignancy has a high tendency to metastasize early and outcome is poor, compared to other pediatric cancers. A systematic protocol and treatment for RT has not yet been established. The incidence of RT is not known, in part because the histological diagnosis is rather difficult before the introduction of a specific antibody. The most established therapy protocol is for RT of the kidney, though treatment variables defining the prognosis have not been clearly identified. In order to create a base for a systematic treatment protocol, we reviewed all the cases of RT in Germany of the 1980s and 1990s for type of manifestation and treatment variables. This analysis aimed at defining prognostic factors for RT and should help to define a treatment schedule including surgery, chemotherapy and radiotherapy.

\section{Patients and methods}

We registered a total of 87 patients with RT in Germany between 1984 and 1999. In 70 patients, there were a follow-up data and written consent available and these patients were included in our study. The source of information was from the German Childhood Cancer Registry in 47 cases, the German Soft Tissue Sarcoma Study in 16 cases and the SIOP-Nephroblastoma database in 24 cases. Analyses were performed by evaluating patient records and having histological slides reviewed by central pathology. Diagnostic criteria for RT were limited to histological features as sufficient material for molecular analysis was not available. Staging of the kidney tumors was performed according to the revised SIOP (International Society of Pediatric Oncology) the staging system (4). For soft tissue tumors, staging system 


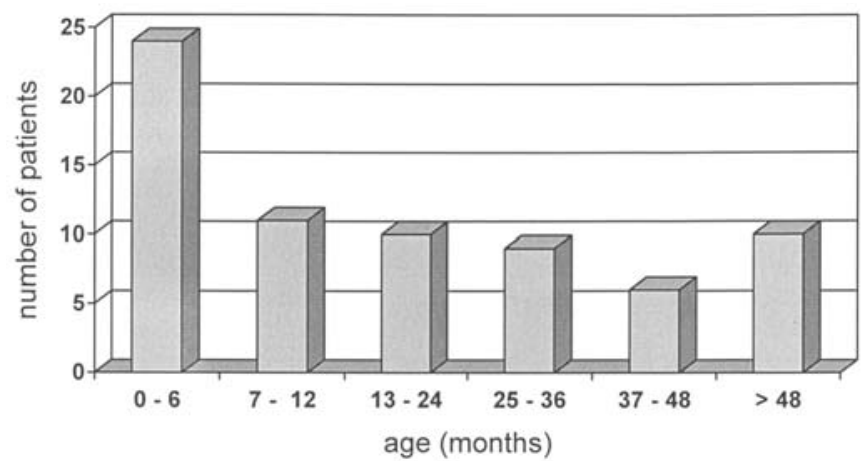

Figure 1. Age distribution of the 70 children with Rhabdoid tumors (1984-1999). The median age is 12 months and the mean age 33 months.

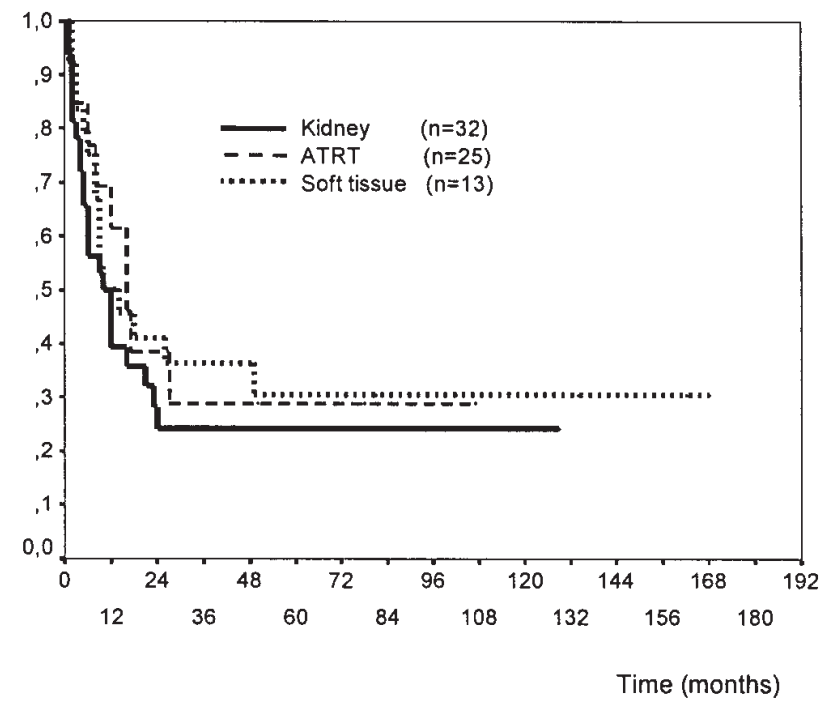

Figure 2. Survival curves of the patients with RT, stratified by localisation of the tumor. There is no difference in outcome between RT of the kidney, AT/RT and soft tissue RT ( $\mathrm{p}=0.64)$.

of the Intergroup Rhabdomyosarcoma Study (IRS) was applied (5).

The overall survival (OS) was measured from the date of initial diagnosis of RT to the date of death or last contact. Survival was estimated according to the Kaplan Meier method for tumor localisation, stage, sex, age and treatment variables (6). Statistical analysis was performed with SPSS software, version 10 .

\section{Results}

Clinical presentation. Thirty-two of the tumors were localized in the kidney, in 25 cases there was a soft tissue tumor in different body regions and in 13 cases an AT/RT was diagnosed. Primary metastases were detected in 18 of the 70 individuals. Of these, 12 had a kidney tumor, 5 a soft tissue manifestation and 1 a cerebral mass. Sites of metastasis were lung $(n=5)$, lymph nodes $(n=8)$, soft tissue $(n=3)$, bone $(n=1)$ and CNS $(n=1)$. Forty-one patients were male and 29 female, giving a male to female ratio of 1.4:1. This ratio was similar for the three localisations of RT. The age at diagnosis of the patients ranged from newborn to older than 4 years with a

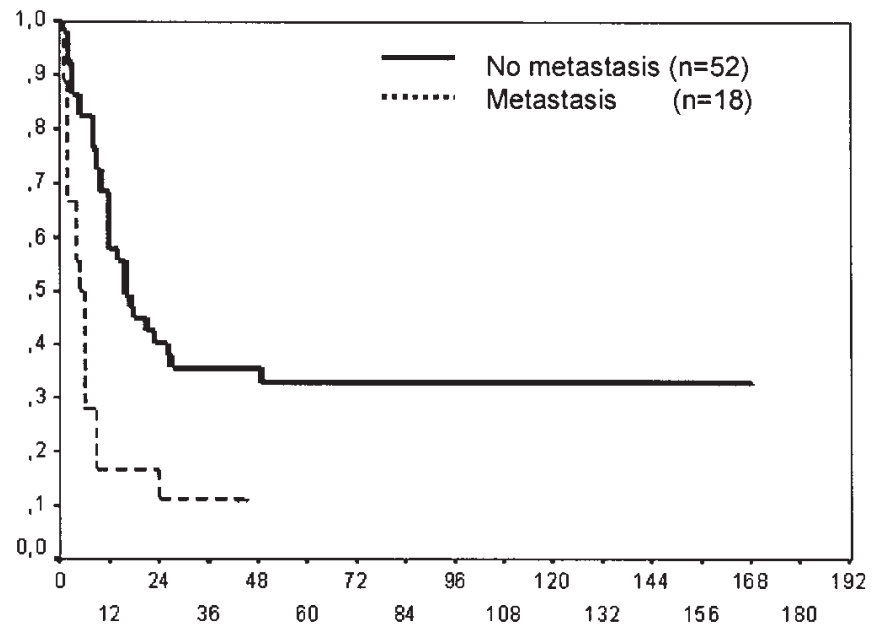

Time (months)

Figure 3. Overall survival (OS) of the children with RT with or without primary metastasis. Presence of metastasis is related to poorer prognosis (0.11) than localised disease $(0.33), \mathrm{p}=0.0003$.

median age of 12 months and a mean age of 33 months (Fig. 1).

After 5 years, overall survival was $27 \%$ for the 70 patients. Males showed a higher survival rate than females with 37 and $17 \%$, respectively $(\mathrm{p}=0.07)$.

Stage and localisation. The 32 patients with $R T$ of the kidney had higher tumor stages and 25 of them were not completely resected (stage III). Five patients had stage I RT and 2 a stage II. Twelve patients had a metastatic disease at diagnosis (stage IV). Soft tissue manifestation of RT was observed in 25 patients. A complete resection with stage I was achieved in 3 cases. Four patients had a stage II and 18 patients showed a stage III. Stage IV was observed in 5 patients. $A T / R T$ could be resected in 4 of the 13 cases, the remaining tumors had incomplete resection or biopsy. Primary metastasis was seen in 1 patient.

OS after 5 years of the patients with RT of the kidney was $24 \%$ and soft tissue manifestation showed $30 \%$ OS and AT/RT of $29 \%$ (Fig. 2). There was no significant difference in survival between these groups $(\mathrm{p}=0.64)$. Patients with initial metastasis had a significantly poorer outcome compared to patients with localised RT (11 vs 33\%, p=0.0003) (Fig. 3). This observation was independent of the site of the primary tumor.

Age at diagnosis. For analysis of age dependence, we stratified the patients according to an age older or younger than one year. The number of patients per group was identical. The 5 -year survival for children younger than one year was $26 \%$, for children older than one year it was $29 \%(\mathrm{p}=0.33)$. For further analysis, we set the cut point at 6 and 24 months of age, though in none of the stratification groups could we see any influence of survival by age. Comparing patients older than 36 months $(n=16)$ with younger children $(n=54)$, we observed a difference in survival (23 vs. $44 \%$ ), although the Kaplan Meier analysis showed no significance ( $\mathrm{p}=0.16$ ) (Fig. 4). 
Table I. Demographics and treatment characteristics of the 70 patients with rhabdoid tumor.

\begin{tabular}{|c|c|c|c|}
\hline & Kidney & Soft tissue & Central nervous system \\
\hline \multicolumn{4}{|l|}{ Gender } \\
\hline Male & 18 & 15 & 8 \\
\hline Female & 14 & 10 & 5 \\
\hline Mean age (months) & 16 & 52 & 37 \\
\hline \multicolumn{4}{|l|}{ Resection } \\
\hline Complete & 11 & 3 & 4 \\
\hline Incomplete & 21 & 22 & 9 \\
\hline \multicolumn{4}{|l|}{ Metastasis } \\
\hline Yes & 12 & 5 & 1 \\
\hline No & 20 & 20 & 12 \\
\hline \multicolumn{4}{|l|}{ Radiotherapy } \\
\hline Yes & 15 & 9 & 4 \\
\hline No & 17 & 16 & 9 \\
\hline \multicolumn{4}{|c|}{ Protocol chemotherapy } \\
\hline Yes & 32 & 24 & 9 \\
\hline No & 0 & 1 & 4 \\
\hline Survival (\%) & 24 & 30 & 29 \\
\hline
\end{tabular}

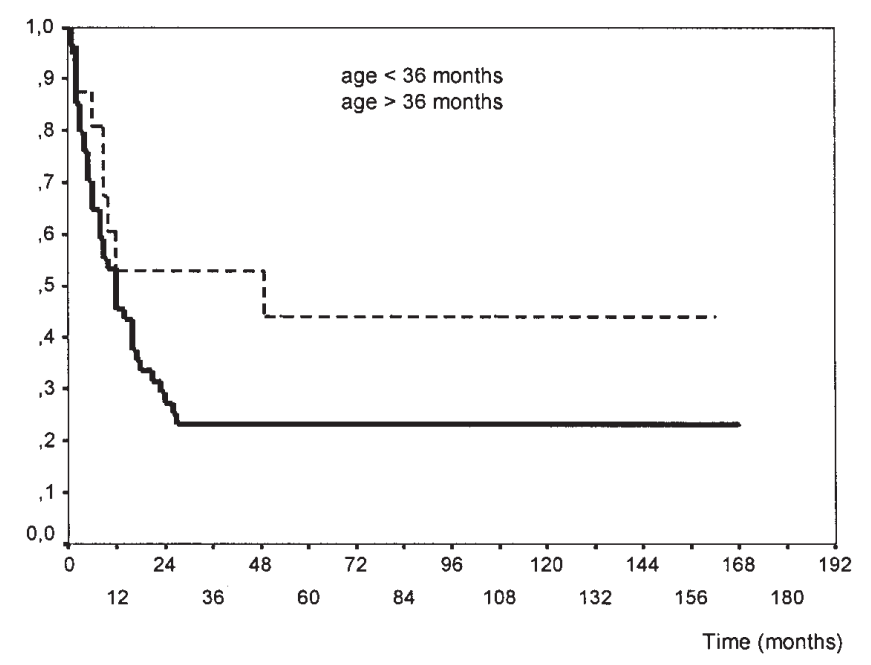

Figure 4. Overall survival of the children with RT by age group. Patients younger than 36 months have a better outcome (0.44) compared to children older than 36 months $(0.24)$, though the log-rank test shows no significance $(\mathrm{p}=0.16)$.

Treatment. Of the 70 registered patients, 65 were included in recent trials of the German Society of Pediatric Oncology and Hematology (GPOH). The 32 patients with RT of the kidney were registered and treated according to SIOP Nephroblastoma Protocols, 24 patients with soft tissue RT were included in sarcoma regimens (CWS; cooperative Weichteilsarkomstudie, CESS; cooperative Ewing Sarcoma Study). AT/RT was treated according to the German brain tumor (HIT) protocols in 9 cases. An individual treatment not according to a clinical trial was applied in 5 children. Demographics and treatment characteristics of the patients are given in Table I.
Surgery. A complete resection of the tumor was achieved in 18 of the 70 patients $(26 \%)$. The remaining children underwent a subtotal resection in 40 cases and a biopsy only in 12 cases. We compared the survival of completely resected with incompletely resected tumors and could not see a significant difference in outcome ( 35 vs. $24 \%, p=0.22$ ). Ten of the 52 patients with a localised disease had complete tumor resection. Their outcome, compared to patients with incomplete resection, was not any better ( 42 vs. $26 \%, \mathrm{p}=0.75$ ). Patients with initial metastasis $(n=18)$ had complete resection in 6 cases, 5 of whom died. Of the 12 patients with metastasis and subtotal resection, only one survived. There is no difference in survival between the two groups $(\mathrm{p}=0.52)$.

Chemotherapy. A total of 65 patients were treated with chemotherapy regimens. As the patients were treated according to the corresponding clinical trials, they received a number of different drugs following the ongoing studies. RT of the kidney was treated with SIOP nephroblastoma high-risk chemotherapy including doxorubicin, carboplatin, cyclophosfamide and etoposide. Patients with soft tissue RT received the VAIA (vincristine, dactinomycin, ifosfamide, adriamycin) regimen in most cases. The brain tumor protocols for AT/RT included cyclophosphamide, methotrexate and etoposide. Due to the diversity of the treatment, the influence of single agents like anthracyclines or alkylating agents could not be evaluated. We formed groups of patients treated according to the same protocol, though we observed no difference in OS for the different groups.

Radiotherapy. Treatment with radiotherapy was applied in 28 of the 70 patients. RT of the kidney was irradiated in $47 \%$, soft tissue RT in $36 \%$ and AT/RT in $30 \%$ of the cases. The OS was 
$29 \%$ among the irradiated patients compared to $25 \%$ in the 42 patients without radiotherapy. Kaplan Meier analysis showed no significant benefit from radiotherapy $(\mathrm{p}=0.50)$. The mean dose of irradiation was 35 Gray. To see the influence of the radiation dose, we compared the 20 patients who received $>30$ Gray to the 8 patients that received $<30$ Gray. This analysis showed no advantage for children receiving a higher radiation dose $(\mathrm{p}=0.40)$. This analysis is biased by the fact of age, since infants did not receive radiotherapy as often as older children. Only $17 \%$ of the patients younger than one year were irradiated, whereas $65 \%$ of the children older than one year received radiotherapy. Furthermore, the different localisations of RT demanded specific radiation strategies respecting the different radiation susceptibility of the adjacent tissue.

\section{Discussion}

Treatment and prognosis of RT in infancy and childhood is still frustrating, compared to other pediatric malignancies. Before the discrimination of RT as an individual entity (1), the tumor was often classified as medulloblastoma, primitive neuroectodermal tumor (PNET) or choroid plexus tumor (CPT) (7). In the kidney, RT was often misdiagnosed as clear cell sarcoma or nephroblastoma. By imaging studies, RT cannot be differentiated from nephroblastoma (8). Only a histological examination can prevent pre-operative treatment according to an inadequate protocol (9). Incidence and biological markers were unknown until molecular analysis revealed changes in chromosome 22 (2) and specific mutations in the gene SMARCB1/hsNF5/INI1 (3). As a uniform treatment strategy for RT is missing, patients are still treated according to different protocols, depending on the site of the tumor manifestation. In order to create a systematic base for further treatment, we retrospectively analysed German RT patients of the 80s and 90s (10).

The incidence of RT is not known. A report from the Automated Childhood Cancer Information System for European children (11) found 45 cases of RT of the kidney between 1988 and 1997. The total number of renal tumors during this time span was 3134 and the number of RT was 45 giving an incidence rate of 0.1 per million children per year. We hypothesize that the real incidence of RT is underestimated in this study. Following the data of our study population, we found 87 cases within 16 years (about 5/year) giving an incidence rate of about 0.5 per million per year. The incidence rate for renal RT alone in our study was 0.23 . The National Wilms Tumor Study Group (NWTS) (12) registered 142 patients with renal RT in North America between 1969 and 2002, thus representing $1.5 \%$ of children with a malignancy of the kidney. A registry for AT/RT has been established and data were published in 2004 (13). Unfortunately, the time span of registration or epidemological data are not mentioned, as the study focuses on treatment and prognostic variables. For RT of the soft tissue, a systematic review was published in 2006 (14), emphasizing the problem of distinguishing RT from soft tissue sarcoma or Ewing's sarcoma. Former publications on soft tissue RT focused on clinicopathological data (15-17) and a large series of patients including clinical data, are not available.
The outcome of patients in this study is poor and is independent of tumor site. Compared to former publications, there was no difference in overall survival. The NWTS report an overall survival of $23.2 \%$ at 4 years for RT of the kidney (12). No differences were observed between males and females, doxorubicin treatment or not, or with or without radiotherapy. A strong correlation was observed for increased survival with increasing age of the children. Higher stages (Stage III and IV) had a significantly poorer prognosis than lower stages. In our population, age at diagnosis was not a significant prognostic parameter, although we observed a trend for a better outcome with increasing age. Tumor stage, especially the presence of metastasis, can be confirmed as a relevant prognostic factor in our study.

For AT/RT, a cohort of 42 patients was published in 2004 (13). The data derived from a newly created registry for RT in the United States. The median survival was 16.75 months and the median event-free survival was 10 months. The authors discuss the possible value of intrathecal chemotherapy, radiotherapy and high-dose chemotherapy. They concluded that multi-institutional and national clinical trials are needed in order to improve the prognosis of AT/RT. The most recent experience with AT/RT patients emphasizes the prognostic relevance of age at diagnosis (18). Children older than 3 years could be cured with a combination of radiation and high-dose alkylating therapy. Infants and younger children had a very poor prognosis with an overall survival of $17 \%$. The authors postulate the importance of tumor control with radiotherapy, as the only long-term survivors in the younger patient cohort had received radiotherapy early in the course of treatment.

The few published patients with RT of the soft tissue have a poor prognosis with a 5-year survival rate of $20 \%$ (14). Within the first year of diagnosis, $42 \%$ of the patients died of the disease.

Our observations of RT in different sites are consistent with former experiences and confirm that the disease still has a very poor prognosis. Future teatment strategies should include primary characteristics of the patients like tumor site, age and stage as stratification criteria. On the other hand, treatment modalities should include the most promising drugs and treatment techniques.

As localisation of the tumor seems to be an independent parameter for outcome, all patients with RT could possibly be treated in a common study. However, this strategy would not take into account the differences of local treatment concerning dosage of radiotherapy and surgical radicality. For age at diagnosis, we saw a trend in favor of older children and the experiences of other studies reveal this parameter as significant $(12,18)$. Therefore, age at diagnosis might be an important stratification criterium for RT patients. The presence of metastasis at diagnosis is the most important prognostic factor in RT. This experience can justify a more aggressive treatment in this subgroup, accepting a higher toxicity of treatment. Chemotherapeutic regimens should include drugs that were formerly regarded as effective like anthracyclines and alkylating agents. The combination of ifosfamide, carboplatinum and etoposide is efficient against RT of the kidney (19) and AT/RT (18). Recent unpublished chemotherapy strategies in North America and Germany prefer intensive multi-drug protocols including topoisomerase I inhibitors in 
combination with the 'classic' drugs. Although there are no studies available for effectiveness as a single drug in RT, topoisomersae I inhibitors could probably play a role in combination treatment. Oxaliplatin has not proven to be effective in patients with refractory Rhabdoid tumors (20). Surgical radicality seems to be of prognostic value in RT of the kidney and AT/RT $(12,19)$. Although in our population, this factor did not show any significant difference in outcome, future strategies should aim to complete surgical removal of the tumor. The role of radiotherapy in the treatment of RT remains controversial. In a systematical review of the literature, Squire et al (21) found an association between initial radiotherapy in AT/RT patients and prolonged survival. However, they emphasize that radiotherapy has its limitations in infants and young children because of severe side effects like endocrine dysfunction, cognitive delay and mental retardation. For RT of the kidney and soft tissue, the role of radiotherapy is difficult to define (12).

Taking into account our own experiences and the data of the literature, a clear concept for the treatment of RT in children is needed. Presently, a cooperative nationwide study is planned for RT patients in Germany.

\section{References}

1. Beckwith JB and Palmer NF: Histopathology and prognosis of Wilms tumors: results from the First National Wilms' Tumor Study. Cancer 1: 1937-1948, 1978.

2. Biegel JA, Rorke LB, Packer RJ and Emanuel BS: Monosomy 22 in rhabdoid or atypical tumors of the brain. J Neurosurg 73: 710-714, 1990.

3. Versteege I, Sevenet N, Lange J, Rousseau-Merck MF, Ambros P, Handgretinger R, Aurias A and Delattre O: Truncating mutations of hSNF5/INI1 in aggressive paediatric cancer. Nature 9: 203-206, 1998.

4. Vujanic GM, Sandstedt B, Harms D, Kelsey A, Leuschner I and de Kraker J: SIOP nephroblastoma scientific committee: Revised international society of paediatric oncology (SIOP) working classification of renal tumors of childhood. Med Pediatr Oncol 38: 79-82, 2002.

5. Raney RB, Anderson JR, Barr FG, Donaldson SS, Pappo AS, Qualman SJ, Wiener ES, Maurer HM and Crist WM: Rhabdomyosarcoma and undifferentiated sarcoma in the first two decades of life: a selective review of intergroup rhabdomyosarcoma study group experience and rationale for Intergroup Rhabdomyosarcoma Study V. J Pediatr Hematol Oncol 23: 215-220, 2001

6. Kaplan EL and Meier P: Nonparametric estimation from incomplete observations. J Am Stat Assoc 54: 457-481, 1958.

7. Reddy AT: Atypical teratoid/rhabdoid tumors of the central nervous system. J Neurooncol 75: 309-313, 2005.

8. Schenk JP, Engelmann D, Rohrschneider W, Zieger B, Semler O, Graf $\mathrm{N}$ and Troger J: Rhabdoid tumors of the kidney in childhood. Rofo 76: 965-971, 2004.
9. Vujanic GM, Kelsey A, Mitchell C, Shannon RS and Gornall P: The role of biopsy in the diagnosis of renal tumors of childhood: Results of the UKCCSG Wilms tumor study 3. Med Pediatr Oncol 40: 18-22, 2003.

10. Kaatsch P: German Childhood Cancer Registry and its favorable setting. Bundesgesundheitsblatt Gesundheitsforschung Gesundheitsschutz 47: 437-443, 2004.

11. Pastore G, Znaor A, Spreafico F, Graf N, Pritchard-Jones K and Steliarova-Foucher E: Malignant renal tumours incidence and survival in European children (1978-1997): report from the Automated childhood cancer information system project. Eur J Cancer 42: 2103-2114, 2006.

12. Tomlinson GE, Breslow NE, Dome J, et al: Rhabdoid tumor of the kidney in the National Wilms' Tumor Study: age at diagnosis as a prognostic factor. J Clin Oncol 23: 7641-7645, 2005.

13. Hilden JM, Meerbaum S, Burger P, Finlay J, Janss A, Scheithauer BW, Walter AW, Rorke LB and Biegel JA: Central nervous system atypical teratoid/rhabdoid tumor: results of therapy in children enrolled in a registry. J Clin Oncol 22: 2877-2884, 2004.

14. Oda Y and Tsuneyoshi M: Extrarenal rhabdoid tumors of soft tissue: clinicopathological and molecular genetic review and distinction from other soft-tissue sarcomas with rhabdoid features. Pathol Int 56: 287-295, 2006.

15. Kodet R, Newton WA Jr, Sachs N, Hamoudi AB, Raney RB, Asmar L and Gehan EA: Rhabdoid tumors of soft tissues: a clinicopathological study of 26 cases enrolled on the Intergroup Rhabdomyosarcoma Study. Hum Pathol 2: 674-684, 1991.

16. Parham DM, Weeks DA and Beckwith JB: The clinicopathological spectrum of putative extrarenal rhabdoid tumors. An analysis of 42 cases studied with immunohistochemistry or electron microscopy. Am J Surg Pathol 18: 1010-1029, 1994.

17. Fanburg-Smith JC, Hengge M, Hengge UR, Smith JS Jr and Miettinen M: Extrarenal rhabdoid tumors of soft tissue: a clinicopathological and immunohistochemical study of 18 cases. Ann Diagn Pathol 2: 351-362, 1998.

18. Tekautz TM, Fuller CE, Blaney S, Fouladi M, Broniscer A, Merchant TE, Krasin M, Dalton J, Hale G, Kun LE, Wallace D, Gilbertson RJ and Gajjar A: Atypical teratoid/rhabdoid tumors (ATRT): improved survival in children 3 years of age and older with radiation therapy and high-dose alkylator-based chemotherapy. J Clin Oncol 23: 1491-1499, 2005.

19. Yamamoto M, Suzuki N, Hatakeyama N, Mizue N, Hori T, Kuroiwa Y, Hareyama M, Oda T, Kudoh T, Nui A, Matsuno T, Hirama T, Yokoyama S, Dome JS and Tsutsumi H: Treatment of stage IV malignant rhabdoid tumor of the kidney (MRTK) with ICE and VDCy: a case report. J Pediatr Hematol Oncol 28: 286-289, 2006.

20. Fouladi M, Blaney SM, Poussaint TY, Freeman BB, McLendon R, Fuller C, Adesina AM, Hancock ML, Danks MK, Stewart C, Boyett JM and Gajjar A: Phase II study of oxaliplatin in children with recurrent or refractory medulloblastoma, supratentorial primitive neuroectodermal tumors and atypical teratoid rhabdoid tumors: a pediatric brain tumor consortium study. Cancer 107: 2291-2297, 2006.

21. Squire SE, Chan MD and Marcus KJ: Atypical teratoid/ rhabdoid tumor: the controversy behind radiation therapy. J Neurooncol 81: 97-111, 2007. 